\title{
General Model and SOC Estimation of Battery
}

\author{
Li Meng \\ College of Electronic Information Engineering, Hebei University, Baoding 071000, Hebei, China \\ Fengfan Co. Ltd, Baoding 071000, Hebei, China. \\ Haipeng Guo \\ Fengfan Co. Ltd, Baoding 071000, Hebei, China. \\ Xiaowei Zhao \\ Fengfan Co. Ltd, Baoding 071000, Hebei, China.
}

Received: June 19, 2020. Revised: February 5, 2021. Accepted: February 27, 2021. Published: March 4, 2021.

\begin{abstract}
Monitoring the battery state is of great importance for the safety and normal of the systems which are powered by batteries. SOC (State of Charge) is one of the most important state parameters of battery. SOC cannot be measured directly. The Kalman filter algorithm is one of the techniques often applied to estimate SOC value. An accurate model is necessary for this algorithm. In this paper, a general SOC model is set up. It takes into account not only the difference between discharging and charging work conditions, but also the influence of the working atmosphere, such as temperature and discharging rate. Then based on this general model, unscented Kalman filter method is used to predict the SOC value. It can avoid the error which is caused by ignoring high-order terms, which is a shortcoming exist in the extended Kalman filter method. The simulation experiments prove the approach can get satisfactory results even when the measurement data is mixed with noise or the initial SOC value is not accurate.
\end{abstract}

Keywords-SOC prediction, general circuit model, Unscented Kalman Filter Method.

\section{INTRODUCTION}

$\mathrm{I}_{\mathrm{s}}^{\mathrm{n}}$ $\mathrm{N}$ modern agriculture, batteries can be find in many places, such as tractors, insecticidal lamps, etc. Over charge or over discharge of battery will cause damage to it, even bring danger to the production. To extend the service life and application safety of battery, it is very important to detect the state of battery. Among all kinds of battery parameters, SOC is of great significance.

SOC can not be measured directly. There are some methods exist to predict the SOC, including the current integral method, open-circuit voltage method, discharge test method. In the laboratory, these methods can accurately obtain the remaining battery power. In practice, the initial SOC value is difficult to get and the current measurement value is always mixed with noise. Often current integral method is not applied separately. When the battery is operating, it is difficult to measure the open circuit voltage directly, and it is not feasible to discharge the battery. There are some improvements based on these two basic methods [1]-[3]. Many intelligent methods are used to estimate the SOC. Neural network [4]-[6] and support vector machine [7] are often chosen. Combined with equivalent model of the battery, some filtering algorithms are applied to estimate the SOC. The commonly used prediction methods are extended Kalman filter (EKF) [8]-[10], unscented Kalman filter (UKF) [11], Particle filter [12] and so on. While an accurate model is essential for these methods. Considering the convenience of model simulation, circuit model is often used [13]-[16].

In this paper, circuit model and UKF method are combined to obtain the SOC of the battery. Because charging and discharging conditions may occur alternately when a battery is working, there is necessity to consider not only the impacts of external temperature and current ratio, but also the influence of charging and discharging processes on the circuit parameters when the circuit model is established. A general model which is suitable for discharging and charging modes is obtained. Based on the model, UKF method is applied, which avoids the error caused by ignoring high-order terms compared with the EKF method.

\section{GENERAL CIRCUIT MODEL}

\section{A. Second-order RC Circuit Model}

Several different kinds of battery models are existed, such as electrochemical model, mathematical model, circuit model and neural network model. The circuit model is easy to implement. In this paper, the RC circuit model is selected and its order is determined to be 2 . This is a compromise of the model accuracy and the calculation complexity. Then some improvements are done to get the general model.

Fig. 1 shows the structure of the RC circuit model.

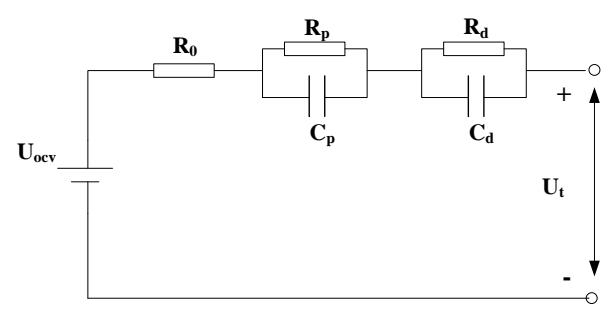


Fig. 1. Second-order RC circuit model

$U_{\mathrm{OCV}}$ represents open circuit voltage. $R_{0}$ is ohmic resistance. $R_{\mathrm{p}}$ and $C_{\mathrm{p}}$ represent the polarization resistance and polarization capacitance of the battery respectively; $R_{\mathrm{d}}$ and $C_{\mathrm{d}}$ respectively represent the diffusion internal resistance and diffusion capacitance of the battery. The circuit parameters are related to the SOC of the battery. For the interesting SOC values, the parameters of circuit model will be identified. The battery which is full of electricity is discharged at a rate of $1 \mathrm{C}$ for 0.1 hours at $25^{\circ} \mathrm{C}$. Then leave it standing for 0.5 hours. Record the SOC value as 0.9. Fig. 2 displays the current flowing through the circuit and terminal voltage during this process.
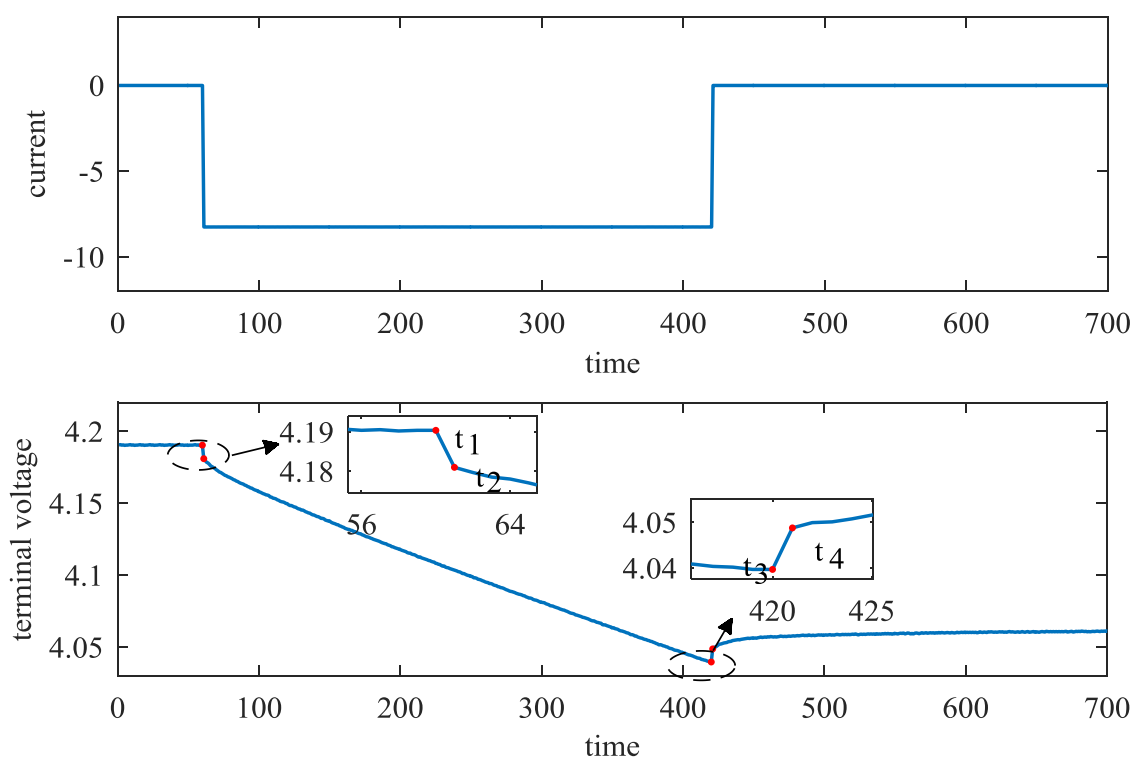

Fig. 2. Experimental data in discharge condition

The discharging current $I$ equals to -8.25 (here, the current is negative when discharging). As shown in the figure, the battery is discharged at time $t_{1}(60 \mathrm{~s})$. At the moment of discharge, because of the action of the ohmic resistor $R_{0}$, the voltage drops from $4.19 \mathrm{~V}$ to $4.18 \mathrm{~V}$ suddenly. From $t_{2}$, the terminal voltage drops steadily. The discharge process lasts $360 \mathrm{~s}$. At $t_{3}(420 \mathrm{~s})$, when the discharge is over, the current becomes zero and the terminal voltage jumps from $4.04 \mathrm{~V}$ to $4.05 \mathrm{~V}$ suddenly. The terminal voltage rises gradually from $t_{4}$, and the rising speed tends to be stable with time. This is caused by the polarization phenomenon of the battery, which is determined by the RC portion in the circuit model. The SOC value of the battery changes when it is discharged, and the corresponding circuit model parameters also change. So the data after discharge is used to identify the circuit model parameters. $R_{0}$ can be obtained by using the amplitude of discharge current and voltage jump at $t_{3}$. The terminal voltage at any time after $t_{4}$ is expressed as (1):

$U(t)=U_{o c v}-I R_{p}\left(e^{-\frac{t-T}{R_{p} C_{p}}}-e^{-\frac{t}{R_{p} C_{p}}}\right)-I R_{d}\left(e^{-\frac{t-T}{R_{d} C_{d}}}-e^{-\frac{t}{R_{d} C_{d}}}\right)$

Where, $\mathrm{T}$ is the time during which the current is acting. $U_{\mathrm{ocv}}$ is open circuit voltage, which can be obtained by data fitting method. In the above experiment, the stationary time is long enough and the terminal voltage can be almost stable, so this stable value is taken as the open circuit voltage. From (1), it can be seen that the relationship between time $t$ and terminal voltage $U(t)$ is non-linear, so nonlinear least square method is applied to obtain electric circuit parameters. Fig. 3 compares the real data with the simulation data obtained from the identified circuit during charging condition. 

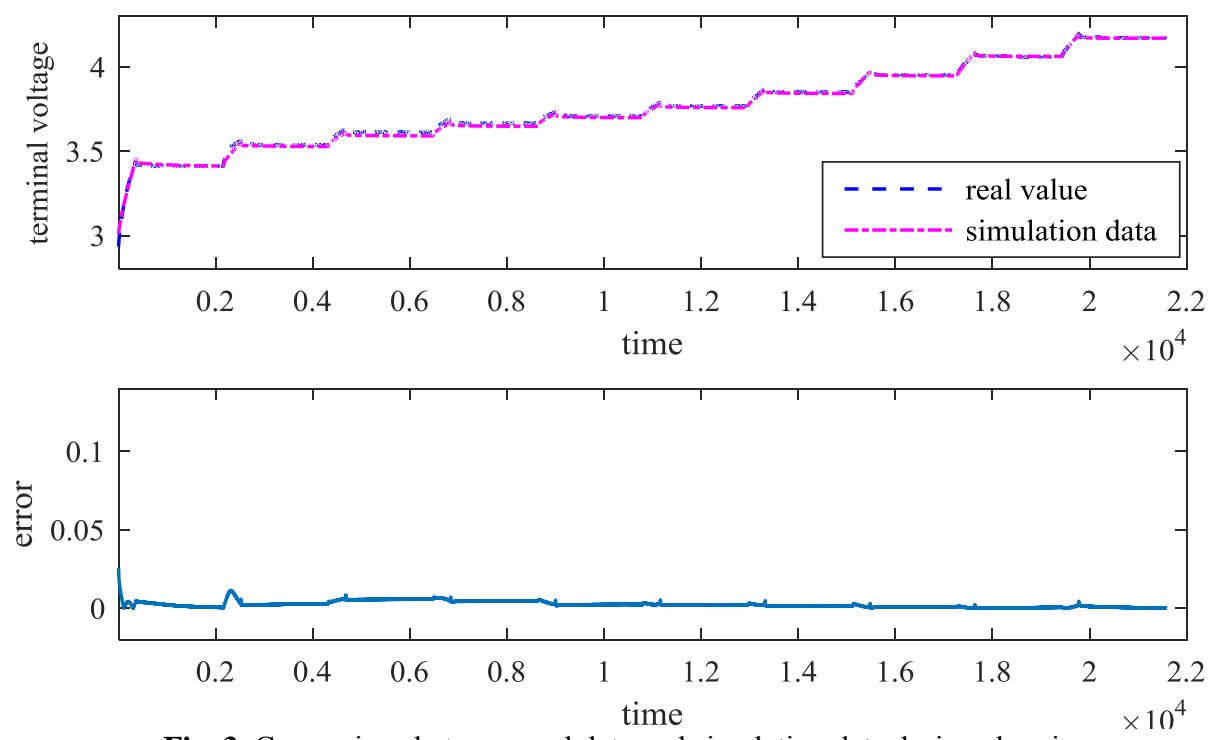

Fig. 3. Comparison between real data and simulation data during charging

The battery has an initial SOC as 0 , that is to say the battery is completely discharged. Then charge the battery ten times, and let it stand for a period of time after each charging. For every charging, the amplitude of the current is equal to $1 \mathrm{C}$, and its action time is 0.1 hour, so the SOC value increases by 0.1 after each charge. From the diagram, we can see that the maximum error between real date and simulation data occurs in the process of the first charge, that is, when the SOC rises from 0 to 0.1 . The errors of other times are very small, almost zero. The discharging condition, which is similar to the charging process, is shown in Fig. 4. A fully charged battery whose initial SOC value is 1.0 is discharged ten times. For every discharging, the amplitude of the current is equal to $1 \mathrm{C}$, and its action time is 0.1 hour, so the SOC value reduces by 0.1 after each charge. At last, the battery is discharged completely. The maximum error between real date and simulation data occurs in the process of the last charge, during which time the SOC drops from 0.1 to 0 . Similarly, the errors of other discharging process are quite near to zero. Thus the identified circuit can better simulate the state of battery when it under charging or discharging condition. Fig. 5 shows the circuit parameters for each SOC value either in discharging mode and charging mode.
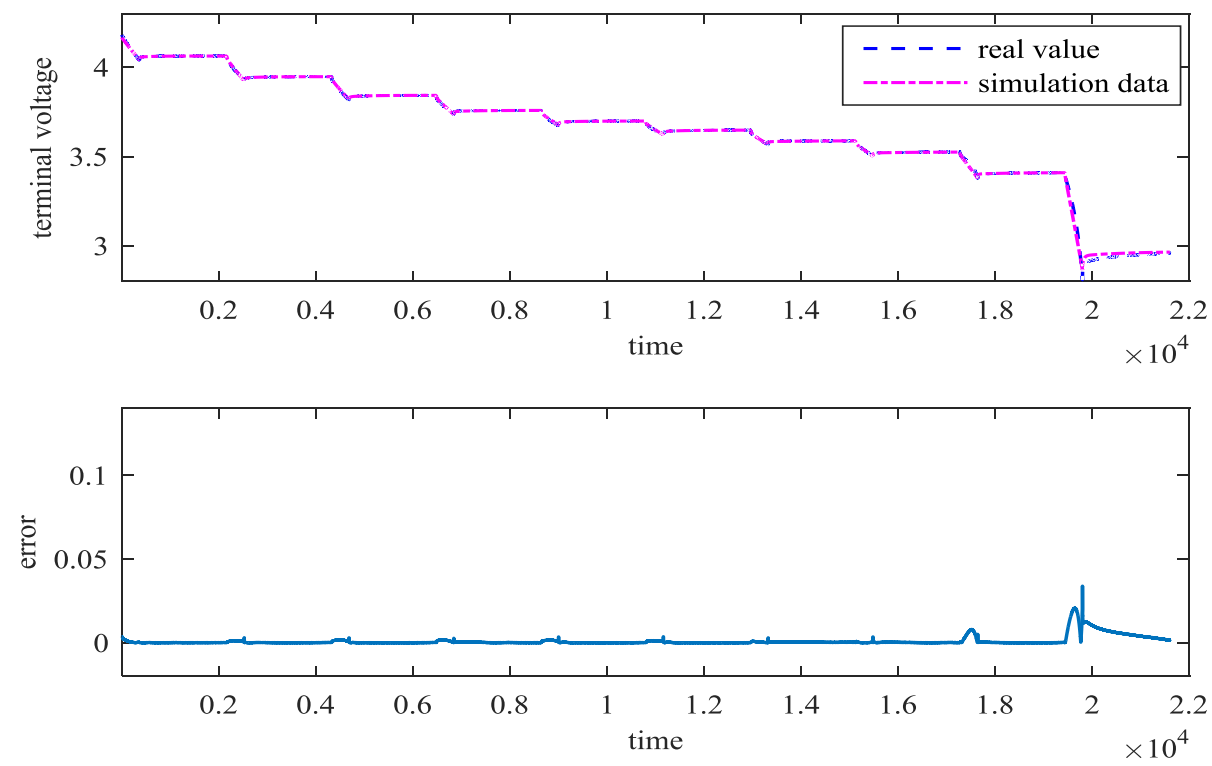

Fig. 4. Comparison between real data and simulation data during discharging 

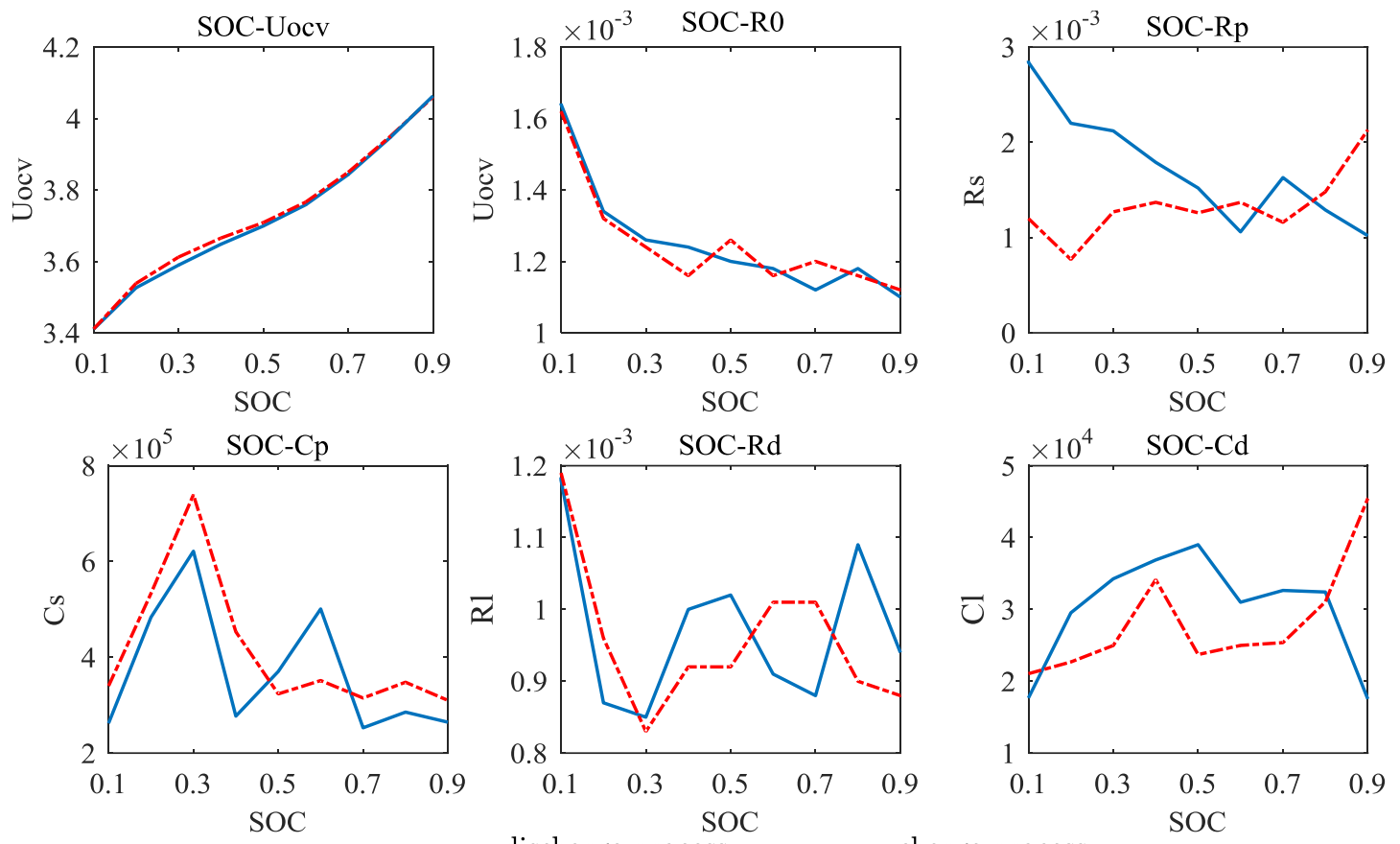

Fig. 5. Circuit model parameters during discharge and charge processes

\section{B. General Model}

Fig. 5 shows that the parameter values of the same circuit element are different for charging and discharging processes. Sometimes the difference is large. However, in many cases, the state of battery charging and discharging can occur alternately. Therefore, it is necessary to consider the universality for charging and discharging processes when modeling the battery Parameters of each circuit element in the original second-order RC circuit shown in Fig. 1 are constant. Based on the original model, some improvements are made to get the general one, which is shown in Fig. 6.

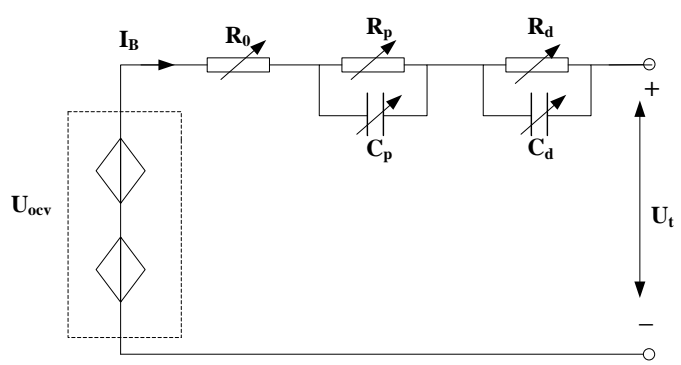

Fig. 6. Improved general Second-order RC circuit model

In the new model, $U_{\text {ocv }}, R_{0}, R_{\mathrm{p}}, C_{\mathrm{p}}, R_{\mathrm{d}}$ and $C_{\mathrm{d}}$ are all variable parameters related to the SOC value and charge and discharge conditions. Take $U_{\mathrm{ocv}}$ as an example. According to subsection A of section II, the identified $U_{\text {ocv }}$ values under different working conditions are shown in Table I and II.

When the current is positive, we calculate the value of $U_{\text {ocv }}$ by linear interpolation method according to Table 1 . That is to say, if the current SOC value falls into [0.3 0.4 , the current value of $U_{\text {ocv }}$ can be obtained by (2)

$$
U_{O C V}=\frac{S O C-0.3}{0.4-0.3}(3.665-3.612)+3.612
$$

Table I. Identified $U_{\text {ocv }}$ under charging condition

\begin{tabular}{cccc}
\hline $\mathrm{SOC} / \%$ & $U_{\mathrm{ocv}} / \mathrm{V}$ & $\mathrm{SOC} / \%$ & $U_{\mathrm{ocv}} / \mathrm{V}$ \\
\hline 0.9 & 4.060 & 0.4 & 3.665 \\
0.8 & 3.950 & 0.3 & 3.612 \\
0.7 & 3.849 & 0.2 & 3.538 \\
0.6 & 3.766 & 0.1 & 3.411 \\
0.5 & 3.708 & & \\
\hline
\end{tabular}

Table II. Identified $U_{\text {ocv }}$ under discharging condition

\begin{tabular}{cccc}
\hline $\mathrm{SOC} / \%$ & $U_{\mathrm{ocv}} / \mathrm{V}$ & $\mathrm{SOC} / \%$ & $U_{\mathrm{ocv}} / \mathrm{V}$ \\
\hline 0.9 & 4.063 & 0.4 & 3.647 \\
0.8 & 3.947 & 0.3 & 3.589 \\
0.7 & 3.843 & 0.2 & 3.526 \\
0.6 & 3.758 & 0.1 & 3.410 \\
0.5 & 3.699 & & \\
\hline
\end{tabular}

Similarly, when the current is negative, Table 2 is employed.

When the initial SOC of batteries is obtained, the SOC value of battery can be calculated by using the ampere-time integral method, which is shown as (3).

$$
z(t)=z(0)-\int_{0}^{t} \frac{i(\tau)}{Q_{n}} d \tau
$$

The parameter value of every element in circuit model is selected according to the SOC value and the current direction. Fig. 7 shows the comparation between real value from battery 
experiment and simulation data from the improved circuit model. We can see that the terminal voltage from general model is very close to the measured data. It should be noted that the current amplitude used in both charging and discharging processes is equal to $1 \mathrm{C}$. As we know, the charge discharge current ratio has an effect on the discharge capacity of the battery. This problem will be taken into account in the next section.
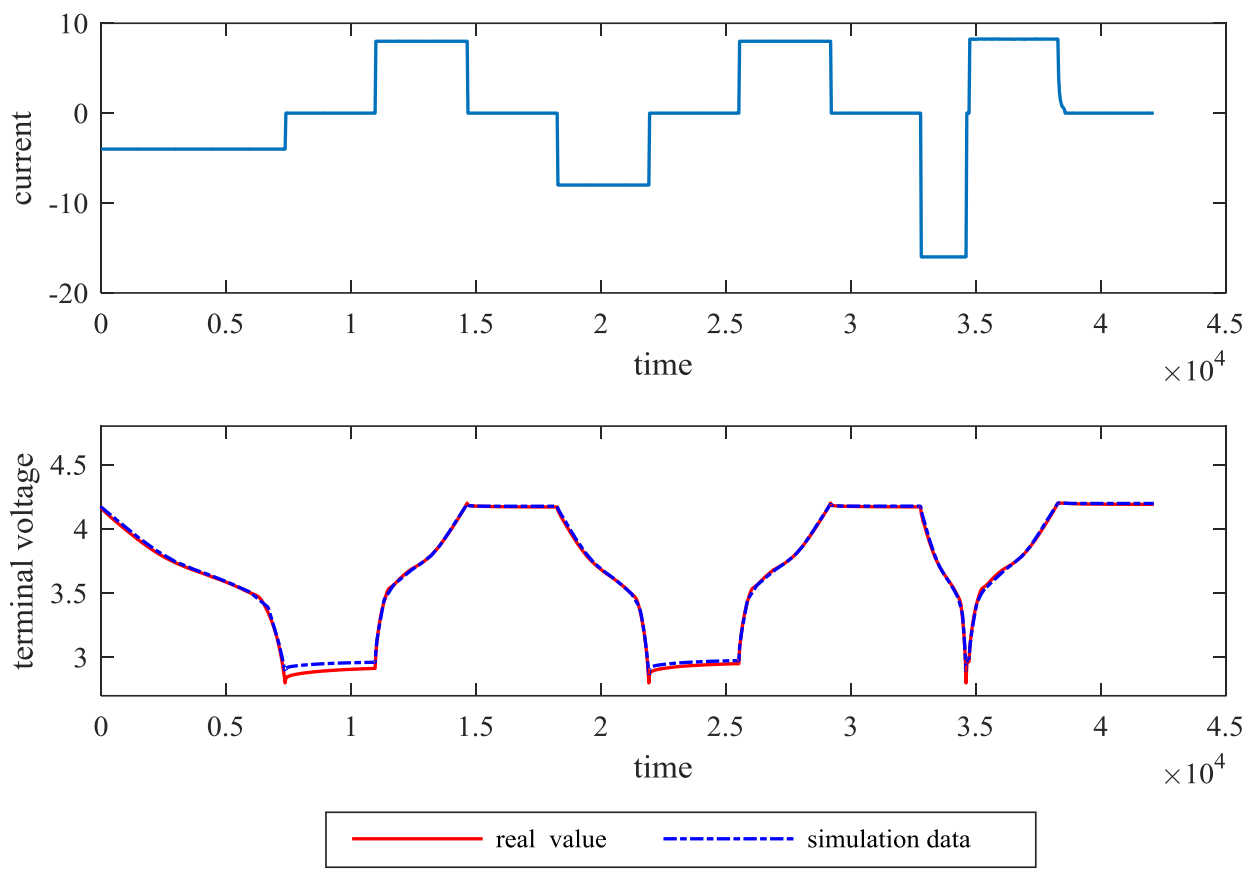

Fig. 7. Model data and measured data during charge and discharge condition

\section{SOC PREDICTION MODEL}

\section{A. Integrated SOC Model}

Let $z(t)$ represent the SOC value at time $t, z(t)$ can be caculated as follows:

$$
z(t)=z(0)-\int_{0}^{t} \frac{\eta i(\tau)}{Q_{n}} d \tau
$$

Where, $z(0)$ is the SOC value at initial time; $i(\tau)$ is the current at time $\tau ; Q_{\mathrm{n}}$ is nominal capacity of the battery; $\eta$ is the coulombic efficiency. In reality, SOC is influenced by discharging rate, the degree aging extent, battery and the external temperature and other factors. Among them, the discharging rate and the temperature have a great influence, so the effect of them on the SOC is integrated into the coefficient $\eta$, as follows:

$$
\eta=\frac{1}{Q_{i} \times Q_{T}}
$$

Where, $Q_{i}$ and $Q_{T}$ represent the impact factors of discharging rate temperature. Take the maximum discharge capacity of a fully charged batter with $1 \mathrm{C}$ at $25^{\circ} \mathrm{C}$ as the standard value, that is equal to 1 . Fig. 8 (a) shows the maximum discharge capacity when a fully charged battery is discharged with 1C at different temperatures. Fig. 8(b) shows the maximum discharge capacity when a fully charged battery is discharged at different discharging rates at $25^{\circ} \mathrm{C}$. As can be seen from the figure, within a certain range, the discharge capacity of the battery increases when the temperature is building up and decreases with the increase of discharge ratio. This is consistent with our common sense.

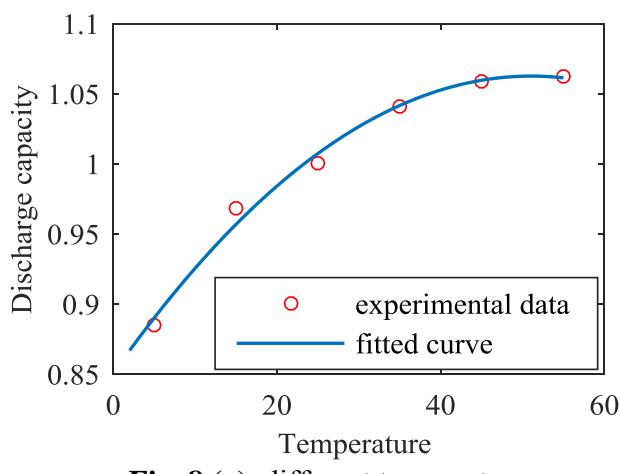

Fig. 8 (a). different temperatures

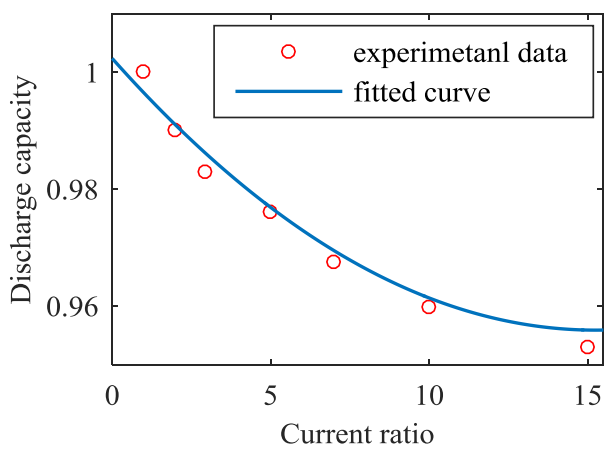

Fig. 8 (b). different discharge rates

Fig. 8. Discharge characteristics of battery 


$$
\begin{aligned}
& Q_{i}=a_{i} C_{i}^{2}+b_{i} C_{i}+c_{i} \\
& Q_{T}=a_{T} T^{2}+b_{T} T+c_{T}
\end{aligned}
$$

The mathematical relation between $Q_{T}$ and discharging rate and that between $Q_{i}$ and temperature can be obtained, show as (6) and (7) respectively. Fit the parameters in Fig. 8(a) and Fig. 8(b) using the least squares method respectively, the coefficients in (6) and (7) are obtained, as flowings:

$$
\left[a_{i}, b_{i}, c_{i}\right]=[0.0002,-0.0061,1.0024] \text {, }
$$$$
\left[a_{T}, b_{T}, c_{T}\right]=[-0.0011,0.0799,-0.2373] \text {. }
$$

\section{B. SOC prediction model}

The state space model of the battery needs to be established for the following prediction work. Considering the parameter measurement problem, the battery charging and discharging current $I$ is taken as the excitation, that is, the input of the system. The battery terminal voltage $U$ is employed as the output of the system. The state variables of the system are three-dimensional. They are SOC value of the battery and voltages of two RC branches, i.e., $U_{p}$ and $U_{d}$ The mathematical relationship between variables in circuit model shown in the battery model can be expressed by (8):

$$
\left\{\begin{array}{c}
\operatorname{SOC}_{k+1}=S O C_{k}-\frac{\eta T}{Q_{N}} I_{k}+w_{1, k} \\
U_{p, k+1}=e^{-\frac{T}{\tau_{p}}} U_{p, k}+\left(1-e^{-\frac{T}{\tau_{p}}}\right) R_{p} I_{k}+w_{2, k} \\
U_{d, k+1}=e^{-\frac{T}{\tau_{d}}} U_{d, k}+\left(1-e^{-\frac{T}{\tau_{d}}}\right) R_{d} I_{k}+w_{3, k} \\
U_{k+1}=U_{o c v, k+1}-I_{k} R_{0}-U_{p, k+1}-U_{d, k+1}+v_{k}
\end{array}\right.
$$

Where, $\tau_{\mathrm{p}}=R_{\mathrm{p}} C_{\mathrm{p}}, \tau_{\mathrm{d}}=R_{\mathrm{d}} C_{\mathrm{d}}$. By writing (8) as a standard state-space expression, it can be obtained as follows:

$$
\left\{\begin{array}{l}
x_{k+1}=A_{k} x_{k}+B_{k} I_{k}+w_{k} \\
y_{k+1}=f\left(S O C_{k+1}\right)-I_{k} R_{0}-U_{p, k+1}-U_{d, k+1}+v_{k}
\end{array}\right.
$$

Where ,

$$
x_{k}=\left[\begin{array}{c}
S O C_{k} \\
U_{p, k} \\
U_{d, k}
\end{array}\right], A=\left[\begin{array}{ccc}
1 & 0 & 0 \\
0 & e^{-\frac{T}{\tau_{p}}} & 0 \\
0 & 0 & e^{-\frac{T}{\tau_{d}}}
\end{array}\right], B=\left[\begin{array}{c}
-\frac{\eta T}{Q_{N}} \\
R_{p}\left(1-e^{-\frac{T}{\tau_{p}}}\right) \\
R_{d}\left(1-e^{-\frac{T}{\tau_{d}}}\right)
\end{array}\right\rfloor
$$

$x_{k}$ is the state space vector, $\mathrm{A}$ is the state transition matrix of the system. B is the input matrix. $w_{k}$ and $v_{k}$ are System noise and measurement noise respectively.

\section{SOC ESTIMATION BASED ON UKF}

\section{A. $U K F$ Algorithm}

In system expressed by (9), SOC is one of the state variable.
The current flowing through the circuit is input of the system. The output of the system is the terminal voltage. The measured data is always with noise. The SOC prediction work is transformed into an optimal estimation of system state based on data containing noise. Kalman filter algorithm can supply an satisfactory solution to such problems. Because the battery system is a complex nonlinear system, the extended filter method is often applied to predicting the value of the battery SOC. In EKF, the system is expressed as the form of Taylor series. Then the higher-order term is omitted to get a linear system approximated to the original system. This brings some error to the estimation of SOC. The UKF approximates nonlinear distribution with sampling method, without linear approximation to the system, and can better fit the nonlinear system. In this paper, UKF algorithm is adopted.

UKF algorithm is a cyclic iterative estimation algorithm. In this algorithm, sigma points are selected according to the distribution characteristic of the reference points. Thus to ensure that the sigma points have the exact same mean value and covariance as $x_{k}$. These sigma points are transferred through the system state function, and the predicted value points can be obtained. Then, the Kalman gain and the error between the real value and the prediction of the observed variables are used to modify the predicted values continuously. At last, the optimal estimation value of the system state variables can be obtained

The operation of UKF to estimate SOC value of battery can be described as follows:

(1) The initialization of the variables, as

$$
\begin{gathered}
x_{0}=E\left[x_{0}\right]=E\left[S O C_{0} \quad 0 \quad 0\right] \\
P=\left[\begin{array}{ccc}
P_{0} & 0 & 0 \\
0 & Q & 0 \\
0 & 0 & R
\end{array}\right]
\end{gathered}
$$

Where, $\mathrm{SOC}_{0}$ stands for the SOC value at the initial time and it is determined by the open circuit voltage method. $P_{0}$ is the estimation variance, $\mathrm{Q}$ is the variance of $w_{\mathrm{k}}$, and $R$ is the variance of $v_{\mathrm{k}}$.

(2) Produce Sigma point at time k:

$$
\begin{gathered}
X_{0}=x_{k} \\
X_{i}=x_{k}+\left(\sqrt{(N+\lambda) P_{k}}\right)_{i}, i=1, \ldots, L \\
X_{i}=x_{k}-\left(\sqrt{(N+\lambda) P_{k}}\right)_{i}, i=N+1, \ldots, 2 N
\end{gathered}
$$

Where, $\lambda=\alpha^{2}(N+k)-N . N$ represents the dimension of the extended state variable, and takes the value of 3 in this system. $\alpha$ is the proportional parameter to determine the expansion of the sigma point around $\mathrm{X}$, and its range is $0 \sim 1$. Here take $\alpha$ as $0.1 . k$ is the scaling parameter, and usually it is equal to 0 in state estimation problem.

(3) Time update. All sigma points are updated backward according to (15), which is another way to write the first equation in (9). 


$$
X_{i, k+1 / k}=f\left(X_{k}, I_{k+1}\right)+w_{k}, i=1, \ldots, 2 N
$$

The prior estimation of the system state can be obtained as:

$$
x_{k+1}^{-}=\sum_{i=0}^{2 N} W_{i}^{m} X_{i, k+1 / k}
$$

Where, weight coefficient $W_{i}^{m}$ are obtained by:

$$
\begin{gathered}
W_{0}^{m}=\frac{\lambda}{\lambda+N} \\
W_{i}^{m}=\frac{\lambda}{2 \lambda+2 N}, i=1, \ldots, 2 N
\end{gathered}
$$

Prior estimation error covariance is:

$$
P_{x, k+1}^{-}=\sum_{i=1}^{2 N} W_{i}^{c}\left(X_{i, k+1 \mid k}-x_{k+1}^{-}\right) \times\left(X_{i, k+1 \mid k}-x_{k+1}^{-}\right)^{T}+Q_{k}
$$

Where, weight coefficient $W_{i}^{c}$ are obtained by:

$$
\begin{aligned}
& W_{0}^{c}=\frac{\lambda}{\lambda+N}+\left(1-\alpha^{2}+\beta\right) \\
& W_{i}^{c}=\frac{\lambda}{2 \lambda+2 N}, i=1, \ldots, 2 N
\end{aligned}
$$

In (20), $\beta$ takes the value of 2 . Its effect is to suppress the error caused by higher-order terms.

(4) Measure updates. Sigma points are propagated backwards according to (22), which is another form of the second equation in (9), to obtain Sigma points of the output variable:

$$
Y_{i, k+1 / k}=g\left(X_{k+1 \mid k}, i_{k+1}\right)+v_{k}
$$

Through UT transformation, the predicted value of measurement estimation is obtained, that is, the mean value of terminal voltage and its covariance are:

$$
\begin{gathered}
y_{k}^{-}=\sum_{i=0}^{2 N} W_{i}^{m} Y_{i, k+1 / k} \\
P_{y, k+1}^{-}=\sum_{i=0}^{2 N} W_{i}^{c}\left(Y_{i, k / k-1}-y_{k}^{-}\right) \times\left(Y_{i, k / k-1}-y_{k}^{-}\right)+R_{k}
\end{gathered}
$$

(5) Modified system state estimation. The joint covariance of the state variable and the output variable at time $k$ is

$$
P_{x y, k+1}^{-}=\sum_{i=0}^{2 N} W_{i}^{c}\left(X_{i, k+1 / k}-x_{k+1}^{-}\right) \times\left(Y_{i, k+1 / k}-y_{k}^{-}\right)^{T}
$$

Kalman filter gain is obtained by (26):

$$
K_{k+1}=P_{x y, k+1}\left(P_{y, k+1}\right)^{-1}
$$

Modify state estimation as following:

$$
x_{k+1}=x_{k+1}^{-}+K_{k+1}\left(y_{k+1}-y_{k+1}^{-}\right)
$$

Modify the state covariance according to (28):

$$
P_{x, k+1}=P_{x, k+1}^{-}-K_{k} P_{y, k+1} K_{k+1}^{T}
$$

(6) Update Noise covariance. The covariance of $w_{k}$ and $v_{k}$ at time $\mathrm{k}$ is calculated by:

$$
\begin{gathered}
Q_{k+1}=K_{k+1} H_{k+1} K_{k+1}^{T} \\
R_{k+1}=H_{k+1}+\sum_{i=1}^{2 N} W_{i}^{c}\left[\left(y_{k+1}\right)_{i}-y_{k}^{-}\right]\left[\left(y_{k}\right)_{i}-y_{k}^{-}\right]^{T} \\
H_{k} \approx \frac{1}{L-1} \sum_{i=k-L+1}^{k} \varepsilon_{i}\left(\varepsilon_{i}\right)^{T}
\end{gathered}
$$

Where, $\varepsilon_{i}$ is the new data of the voltage of the battery model. $H_{k}$ is the approximation of the covariance of $\varepsilon_{i}$.

\section{B. Simulation Experiment}

The experiment data of current and terminal voltage is displayed in Fig. 9. As shown in the diagram, the measured current data and terminal voltage data are mixed with noise. This is consistent with the real site environment. To predict the SOC value of the battery, the SOC value at initial time should be known. The SOC initial value is usually obtained by the open circuit voltage method or by the record value at the previous time. Its accuracy is worth considering. So in the following experiments, two cases are covers: the initial SOC has a correct value or incorrect value. The real value of initial $\mathrm{SOC}$ is 0.7 When the initial SOC value is correct, that is to say, the initial values for ampere hour integration method and UKF method are also 0.7 , the SOC results obtained by ampere hour integration method and UKF method are shown in Fig. 10 respectively. In the beginning, the ampere hour integration method is more accurate, but with the increase of time, the accumulated error of the current increases. Therefore, SOC obtained by ampere-hour integration method is increasingly deviated from the true value. On the contrary, because of the initial of parameters in UKF algorithm, in the first 800 seconds, UKF estimation error is relatively larger than that obtained by ampere hour integration method. While it is getting closer to the real value along with time. Especially after 1500 seconds, it almost coincides with the real value. Fig. 11 display the results when the SOC value at initial time is incorrect. The actual value of SOC is 0.8 , when the initial value is incorrectly estimated as 0.7 , the ampere hour integration method can not avoid the impact of the initial value estimation error. After several iterations, UKF can track the true value well.

This is because when the UKF method is applied to predict battery SOC value, after the state variable is initialized, it updates the SOC value when the new sampling data is obtained. At the same time, according to the error covariance, the Kalman gain is modified in a timely manner, and the estimation error will be corrected by feedback. With the accumulation of time, the number of cycles increases, and the SOC estimation value keeps approaching the real value. UKF algorithm is capable of correction in prediction and estimation. Even if the initial value is far from the real value, the predicted value can gradually get close to the true value along with the algorithm process. So UKF is not sensitive to the initial value. This feature makes the algorithm very advantageous in practical application. 

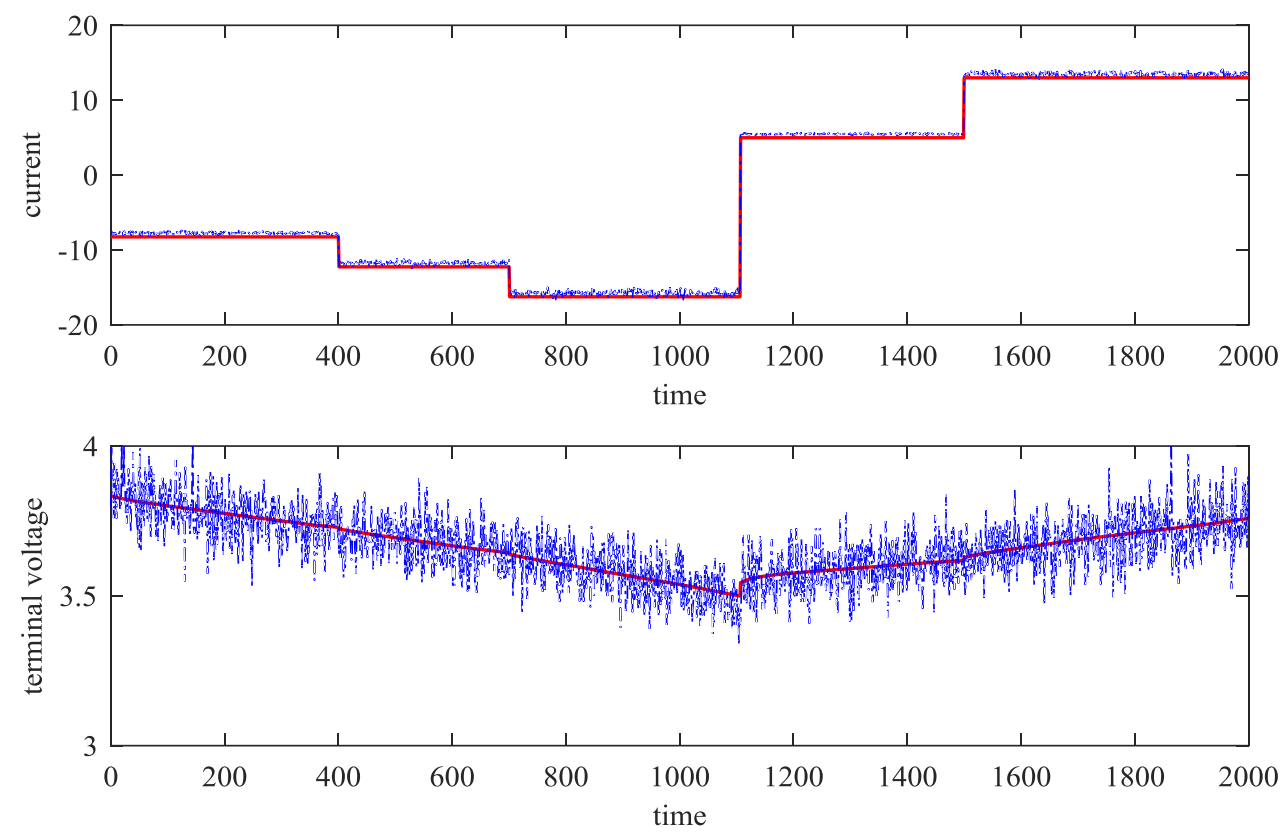

$\longrightarrow$ true value $-\cdot \cdot \cdot-\cdot-\cdot \cdot$ measured value

Fig. 9. Current and terminal voltage during process

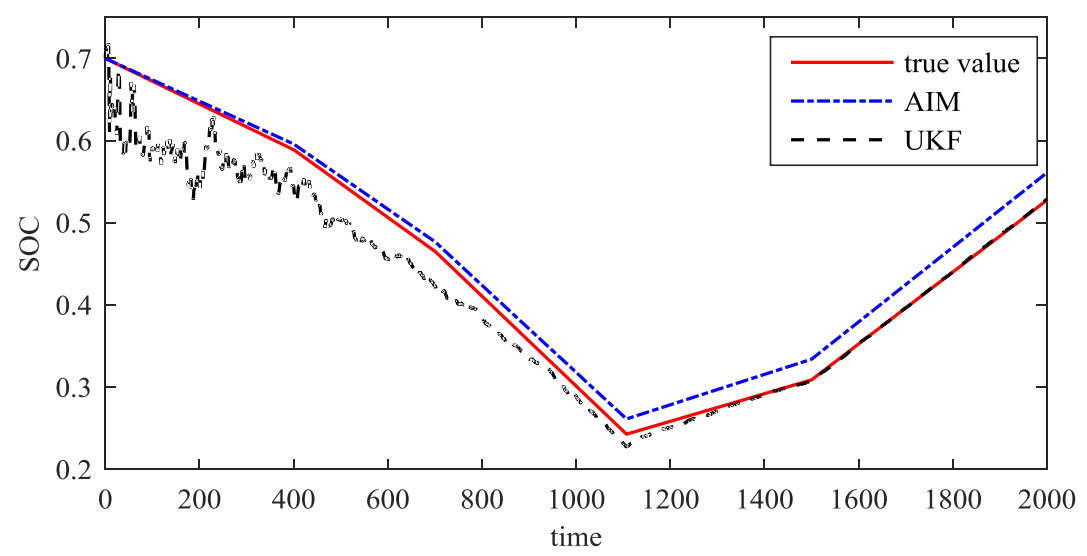

Fig. 10. SOC estimation results with correct initial value

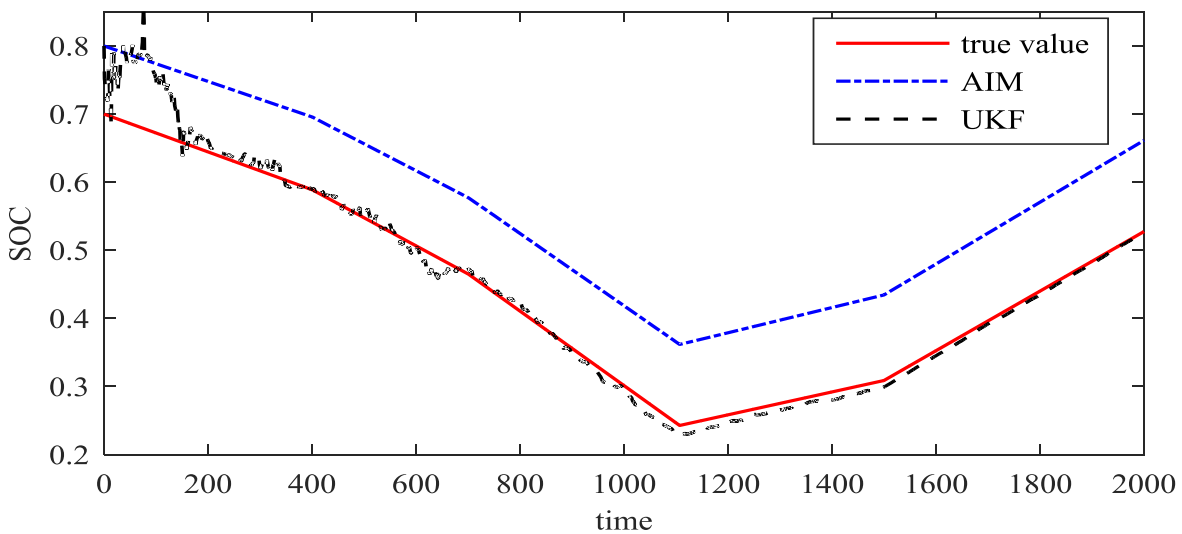

Fig. 11. SOC estimation results with incorrect initial value 


\section{CONCLUSIONS}

In this paper, a general circuit model for battery is built, which is suitable for both charging and discharging conditions. The effects of current rate and temperature are integrated into the calculation formula of SOC. So a complete and comprehensive SOC computing model can be obtained. Then UKF algorithm is applied to estimate the SOC value. The experiment results prove that the combination of general model and UKF can have a satisfactory prediction. The accurate estimation of the state of the battery can ensure its normal operation.

\section{ACKNOWLEDGMENT}

This work was supported in part by a grant from Advanced Programs of Hebei Postdoctoral (Grant No. B2019003027).

\section{REFERENCES}

[1] C. H. Lee, M. Y. Chen, S. H. Hsu, and J. A. Jiang. "Implementation of an SOC-based four-stage constant current charger for Li-ion batteries", Journal of Energy Storage, vol.18, pp. 528-537, 2018.

[2] F. Claude, M. Becherif, and H. S. Ramadan, "Experimental validation for Li-ion battery modeling using Extended Kalman Filters", International Journal of Hydrogen Energy, vol. 42, no.40, pp. 25509-25517, 2017

[3] X. Xiong, S.L. Wang, C. Fernandez, C.M. Yu, C.Y. Zou and C. Jiang, "A novel practical state of charge estimation method: an adaptive improved ampere-hour method based on composite correction factor", International Journal of Energy Research, vol. 44, no.14, pp. 11385-11404, 2020

[4] M. Talha, F. Asghar, and S. H. Kim, "A Neural Network-Based Robust Online SOC and SOH Estimation for Sealed Lead-Acid Batteries in Renewable Systems", Arabian Journal for Science and Engineering, vol. 44, no.3, pp. 1869-1881, 2019.

[5] A. G. Kashkooli, H. Fathiannasab, Z. Y. Mao, and Z. W. Chen, "Application of Artificial Intelligence to State-of-Charge and State-of-Health Estimation of Calendar-Aged Lithium-Ion Pouch Cells", Journal of the Electrochemical Society, vol. 166, no.4, pp. A605-A615, 2019.

[6] E. Chemali, P. J. Kollmeyer, M. Preindl, R. Ahmed, and A. Emadi, "Long Short-Term Memory Networks for Accurate State-of-Charge Estimation of Li-ion Batteries", IEEE Transactions on Industrial Electronics, vol. 65, no.8, pp. 6730-6739, 2018.

[7] T. Zahid, K. Xu, W. M. Li, C. M. Li, and H. Z. Li, "State of charge estimation for electric vehicle power battery using advanced machine learning algorithm under diversified drive cycles", Energy, vol. 162, pp. 871-882, 2018.

[8] C. Chen, R. Xiong, R. X. Yang, W. X. Shen, and F. C. Sun, "State-of-charge estimation of lithium-ion battery using an improved neural network model and extended Kalman filter", Journal of Cleaner Production, vol. 234, pp. 1153-1164, 2019.

[9] C. Jiang, S.L. Wang, B. Wu, B. Etse-Dabu, and X. Xiong, "A Novel Adaptive Extended Kalman Filtering and Electrochemical-Circuit Combined Modeling Method for the Online Ternary Battery state-of-charge Estimation", International Journal of Electrochemical Science, vol. 10, no.10, pp. 9720-9733, 2020.

[10] Y. Qiu, W. Chen, Z. M. Duan, and L. Yu, "State of charge estimation of vanadium redox battery based on improved extended Kalman filter", Isa Transactions, vol. 94, pp. 326-337, 2019.

[11] M. S. El Din, A. A. Hussein, and M. F. Abdel-Hafez, "Improved Battery SOC Estimation Accuracy Using a Modified UKF With an Adaptive Cell Model Under Real EV Operating Conditions", IEEE Transactions on Transportation Electrification, vol. 4, no.2, pp. 408-417, 2018.

[12] Z. H. Chen, H. Sun, G. Z. Dong, J. W. Wei, and J. Wu, "Particle filter-based state-of-charge estimation and remaining-dischargeable-time prediction method for lithium-ion batteries", Journal of Power sources, vol. 414, pp. 158-166, 2019.
[13] S. Ghose, A. El-Shahat, "Modelling and Simulation of Grid Connected Lithium-ion Battery Using HOMER", WSEAS Transactions on Circuits and Systems, Vol. 16, pp. 181-186, 2017.

[14] K. Wang, X. Feng, J. B. Pang, J. Ren, C. X. Duan and L. W. Li, "State of Charge (SOC) Estimation of Lithium-ion Battery Based on Adaptive Square Root Unscented Kalman Filter", International Journal of Electrochemical Science, vol. 15, no.9, pp. 9499-9516, 2020.

[15] W.Q. Li, Y. Yang, D.Q. Wang and S.Q. YIN, "The multi-innovation extended Kalman filter algorithm for battery SOC estimation", Ionics, vol. 26, no.12, pp. 6145-6156, 2020.

[16] M. Gholizadeh and A. Yazdizadeh, "Systematic mixed adaptive observer and EKF approach to estimate SOC and $\mathrm{SOH}$ of lithium-ion battery", IET Electrical systems in transportation, vol. 10, no.2, pp. $135-143,2020$.

Li Meng was born on Jul. 13, 1985. She received the PhD degree in Control theory and Control Engineering from North China Electric Power Universiyt. Currently, he is a researcher (Lecturer) at Hebei University, China. Her major research interests include system identification, intelligent optimization algorithms and intelligent control.

Haipeng Guo was born on Oct. 12, 1982. He received the Master Degree in materials physics and chemistry from Beijing University of Chemical Technology. Currently, he is a Senior Engineer at FengFan Co., LTD, China. His major research interests include product and process of LIB and other advanced battery.

Xiaowei Zhao was born on March 28, 1991. He received the Master's degree in Chemical analysis from Hebei University of China. Currently, he is a Lithium battery engineer at Fengfan Co., Ltd. His main research is Lithium Battery Simulation, Material development, Lithium cell design .etc.

\section{Creative Commons Attribution License 4.0 (Attribution 4.0 International, CC BY 4.0)}

This article is published under the terms of the Creative Commons Attribution License 4.0 https://creativecommons.org/licenses/by/4.0/deed.en_US 